\title{
Damage Identification of High-speed Maglev Guideway Girder Based on Modal Identification
}

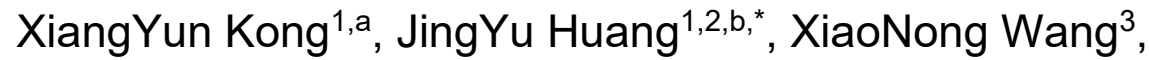 \\ ShuoWei Wang ${ }^{1}$, Liang Zhao ${ }^{1}$, ZhiHong Fang ${ }^{1}$ \\ ${ }^{1}$ Department of Civiling-Engineering, Tongji University, Shanghai, China \\ 2 National Maglev Transportation Engineering R\&D Center, Tongji University, Shanghai, China \\ ${ }^{3}$ Department of Transportation Engineering, Tongji University, Shanghai, China \\ akongxiangyun@tongji.edu.cn, bhuangjingyu@tongji.edu.cn
}

\begin{abstract}
Keywords: High-Speed Maglev, Modal Identification, Guideway Girder, Dynamic Response, Damage Identification
\end{abstract}

\begin{abstract}
As a modern high-tech rail vehicle, the maglev train realizes the non-contact suspension and guidance between the train and the guideway, which greatly reduces the resistance of the system. Due to the high-speed operation characteristics of maglev trains, the structural health monitoring of guideway girders is particularly important for the safety and stability of maglev train operation. This paper takes the maglev train guideway girder as the monitoring target, and the finite element model of the maglev vehicle-guideway is established to simulate the running state of the train passing through the guideway girder. The dynamic response data of the guideway girder is obtained in the finite element model, considering healthy states and different damage states of the guideway girder. Then, a modal-based damage identification method is proposed, which obtains the guideway girder damage sensitive characteristics by decomposing the guideway girder acceleration response signal. Finally, based on the measured guideway girder acceleration data, this paper verifies the effectiveness of the damage identification method in guideway girder structure health monitoring, which provides reference and guidance for the future maintenance of the maglev guideway girder.
\end{abstract}

\section{Introduction}

The $600 \mathrm{~km} / \mathrm{h}$ high-speed maglev has an irreplaceable role in the transportation of the core city circle. At present, relevant theoretical knowledge and experimental researches are being vigorously carried out. With the continuous improvement of vehicle speed and carrying capacity, the structural health of the high-speed maglev track has become more prominent in operation and maintenance.

Current maglevs are often overhauled based on operating mileage or operating years, however track damage cannot be found in time, and maintenance costs and time costs are high. The structural health monitoring system can provide real-time warning when the track is damaged [1-3], accurately locate the damaged part[4], and ensure driving safety and stability. However, there are few researches on structural health monitoring systems related to maglev rails, especially high-speed maglev rails.

The idea of wavelet theory adopted in this paper was formed at the beginning of last century. The Haar system proposed by Haar is the first orthogonal basis of wavelet specification. In 1980, French mathematician Morlet proposed the wavelet transform. Morlet and French theoretical physicist Grossman jointly proposed several systems of continuous wavelet transform, which are based on invariance under translation and contraction. This immutability allows a signal to be 
decomposed according to its independent contributions to space (time) and scale (frequency), It is particularly important that the transform do not lose the original information. Using wavelet transform to identify modal parameters, Firstly, based on the good time-frequency analysis capability of the frequency modulated Gaussian wavelet transform and the band pass filtering property, the system is automatically decoupled. Then the modal parameters are identified from the wavelet transform of the impulse response function. Continuous wavelet transform method for structural modal parameter identification is a new direction in the field of modal analysis, which was first published by Staszewski in 1997[5]. This method takes advantage of the attenuation characteristic of wavelet function, which is similar to the attenuation of structural system's free response signal in form. A clear ridge line can be formed in the time-scale (time-frequency) plane by continuous wavelet transform of free response signal based on stationary phase theory. By extracting the wavelet transform coefficient of the ridge line, the modal parameters of the system can be separated. Ruzzene et al[6] used this method to identify the free response parameters of the multi-degree of freedom system, and pointed out that this method was superior to Hilbert transform in instantaneous frequency identification. Scholars have studied the damping, natural frequency and mode identification of linear systems [7-12].For the parameter identification of nonlinear system, Staszewski adopts the concept of instantaneous phase to identify the instantaneous frequency and amplitude variation of the system[13]. Yonggang Wang and Jinghua Zhang adopted a different wavelet operation [14] and derivation process from Staszewski to study the extraction of modal parameters and derivation of related theories focusing on linear structural systems. For the selection of wavelet transform parent wavelet, Morlet wavelet was used at the beginning, then Cauchy wavelet [9] and Harmonic wavelet line[7] used in mathematical operations. Simonovski [15] made a comparative study of multiple parent wavelets and obtained the advantages of Morlet wavelet for modal parameter identification.

In summary, the wavelet transform method of modal analysis is a new method of modal parameter identification, which is gradually attracting international attention. The direction of the research problem has been in-depth from the identification of modal parameters such as damping, natural frequency, and mode of linear multi-degree-of-freedom systems to the parameter identification of nonlinear systems.

\section{Wavelet Transform Theory}

Set $\psi_{a, b}(t)=\frac{1}{\sqrt{|a|}}=\psi\left(\frac{t-b}{a}\right), a \neq 0, b \in R$, in which $a$ presents the stretch factor (scale factor), and $b$ represents the translation parameters. $\psi_{a, b}(t)$ is a wavelet function which depends on parameter $a$ and $b$. Since $a$ and $b$ are continuous value, the corresponding function wavelet basis function family $\left\{\psi_{a, b}(t)\right\}$ is called the continuous wavelet basis function. Let $\psi(t)$ be a wavelet function, the continuous wavelet transform is defined as formula (1), and the corresponding frequency domain is expressed as formula (2).

$$
\begin{aligned}
& W T_{\psi} f(a, b)=\frac{1}{\sqrt{|a|}} \int_{-\infty}^{+\infty} f(t) \overline{\psi\left(\frac{t-b}{a}\right)} d t \\
& \hat{\psi}_{a, b}(w)=\frac{\sqrt{a}}{2 \pi} \int_{-\infty}^{+\infty} X(w) \hat{\psi}(a w) e^{j w b} d w
\end{aligned}
$$


Let the window radius of the wavelet function $\psi(t)$ be $\Delta t$, and center be $t_{0}$. Then the window radius of its Fourier transform $\hat{\psi}(w)$ is $\Delta w$, and center is $w_{0}$. The relational expressions of $t_{0}$ and $w_{0}$ are as formulas(3) and formulas (4), while the relational expressions of $\Delta t, \Delta w$ and $w_{0}$ are as formulas (5) and formulas (6).

$$
\begin{aligned}
& t_{0}=\frac{1}{\|\psi\|_{2}} \int_{-\infty}^{+\infty} t|\psi(t)|^{2} d t \\
& w_{0}=\frac{1}{\|\hat{\psi}\|_{2}} \int_{-\infty}^{+\infty} w|\hat{\psi}(w)|^{2} d w \\
& \Delta t=\frac{1}{\|\psi\|_{2}}\left[\int_{-\infty}^{+\infty}\left(t-t_{0}\right)^{2}|\psi(t)|^{2} d t\right]^{\frac{1}{2}} . \\
& \Delta w=\frac{1}{\|\hat{\psi}\|}\left[\int_{-\infty}^{+\infty}\left(w-w_{0}\right)^{2}|\hat{\psi}(w)|^{2} d w\right]^{\frac{1}{2}}
\end{aligned}
$$

The center of the window of $\psi_{a, b}(t)$ is $t_{a, b}=a t_{0}+b$ and the width is $\Delta t_{a, b}=a \Delta t$. The center of the window of $\hat{\psi}_{a, b}(w)$ is $w_{a, b}=\frac{1}{a} w_{0}$, and the width is $\Delta w_{a, b}=\frac{1}{a} \Delta w_{0}$. The wavelet coefficient where the function $f(t)$ at a certain scale factor $a$ and translation parameter $b$ represents the size of the frequency components in the frequency window with the center frequency of $\frac{w_{0}}{a}$ and the width of $2 \frac{w_{0}}{a}$ in the time period $2 a \Delta t$ at the position $b$. When the function $\psi(t)$ 满satisfies the allowable condition of formula (7), the inverse transformation of continuous wavelet transform is as formula (8).

$$
\begin{aligned}
& C_{\psi}=\int_{-\infty}^{+\infty} \frac{|\hat{\psi}(w)|^{2}}{|w|} d w<\infty \\
& f(t)=\frac{1}{C_{\psi}} \int_{-\infty}^{+\infty} \frac{1}{a^{2}} d a \int_{-\infty}^{+\infty} W T_{\psi} f(a, b) \psi_{(a, b)}(t) d b
\end{aligned}
$$

As the wavelet generated by the base wavelet $\psi_{a, b}(t)$ acts as an observation window for the signal being analyzed in the wavelet transform, $\psi(t)$ satisfies the constraint condition of the general function, as shown in formula (9). 


$$
\int_{-\infty}^{+\infty}|\psi(t)| d t<\infty
$$

\section{Modal Parameter Identification And Selection of Wavelet Function}

Basic principle of modal parameter identification based on Wavelet Transform

Suppose the signal to be identified is $x(t)=A(t) \cos (w t)$. The analytic signal obtained by Hilbert transform is formula (10), where $H[x(t)]$ is the Hilbert transform of $x(t)$, as in formula (11).

$$
\begin{aligned}
& x_{a}(t)=x(t)+j H[x(t)]=A(t) e^{j w t} . \\
& H[x(t)]=x(t) * \frac{1}{\pi t}=\frac{1}{\pi} \int_{-\infty}^{+\infty} x(\tau) \frac{1}{t-\tau} d \tau .
\end{aligned}
$$

The analytic signal after Hilbert transform has the same amplitude and frequency range as the original signal, which contains the phase information of the original data. The wavelet transform relationship between signal $x(t)$ and its analytical signal $x_{a}(t)$ is shown in formula (12), and the specific analytical formula is shown in formula (13).

$$
\begin{aligned}
& \mathcal{W}_{x}(a, b)=\frac{1}{2} \mathcal{w}_{x_{a}}(a, b) \\
& \mathcal{W}_{\psi}(a, b)=\left\langle x(t), \psi_{a, b}(t)\right\rangle=\frac{1}{2}\left\langle x_{a}(t), \psi_{a, b}(t)\right\rangle=\frac{1}{2 \sqrt{a}} \int_{-\infty}^{+\infty} A(t) e^{j w t} \psi^{*}\left(\frac{t-b}{a}\right) d t
\end{aligned}
$$

Since the wavelet function $\psi_{a, b}(t)$ has compact support, the Taylor series of $A(t)$ near $t=b$ can be expanded as formula (14).

$$
\mathcal{W}_{\psi}(a, b)=\left\langle x(t), \psi_{a, b}(t)\right\rangle=\frac{1}{2}\left\langle x_{a}(t), \psi_{a, b}(t)\right\rangle=\frac{1}{2 \sqrt{a}} \int_{-\infty}^{+\infty}[A(b)+o(A(b))] e^{j w t} \psi^{*}\left(\frac{t-b}{a}\right) d t
$$

Omitting the higher order terms, we get the following results:

$$
\mathcal{W}_{\psi}(a, b)=\left\langle x(t), \psi_{a, b}(t)\right\rangle=\frac{1}{2}\left\langle\boldsymbol{x}_{a}(t), \psi_{a, b}(t)\right\rangle=\frac{\sqrt{a}}{2} A(b) e^{j w b} \psi^{*}(a w)
$$

\section{System modal parameter identification}

The expression of the impulse response function of single-degree-of-freedom viscous damping system is shown in formula (16), where $A_{0}$ is the vibration amplitude. $\mathcal{W}_{d}$ and $\mathcal{W}_{n}$ are respectively the pi ratio of the system without damping and with damping, and $w_{d}=\sqrt{1-\xi^{2}} w_{n} ; \xi$ is the damping ratio. $\varphi_{0}$ represents the initial phase.

$$
x(t)=A_{0} e^{-\xi w_{n} t} \cos \left(w_{d} t+\varphi_{0}\right) .
$$


The wavelet transform of impulse response function of single-degree-of-freedom viscous damping system is shown in formula (17), then the modulus and phase of wavelet transform coefficients are shown in formula (18). The relationship between damping and frequency of transformed system is shown in formula (19).

$$
\begin{aligned}
& w_{x}(a, b)=\frac{\sqrt{a}}{2} A_{0} e^{j\left(w_{d} b+\varphi_{0}\right)} \psi^{*}\left(a_{w_{d}}\right) e^{-\xi w_{n} b} \\
& \left\{\begin{array}{l}
\left|w_{x}(a, b)\right|=\frac{\sqrt{a}}{2} A_{0} e^{-\xi w_{n} b}\left|\psi^{*}\left(a_{w_{d}}\right)\right| \\
\arg \left(w_{x}(a, b)\right)=w_{d} b+\varphi_{0}
\end{array}\right. \\
& \left\{\begin{array}{l}
\xi_{w_{n}}=-\frac{\mathrm{d}}{\mathrm{d} b}\left(\ln \left|w_{x}(a, b)\right|\right) \\
w_{d}=\frac{\mathrm{d}}{\mathrm{d} b}\left(\arg \left(w_{x}(a, b)\right)\right)
\end{array}\right.
\end{aligned}
$$

The expression of impulse response function of multi-degree-of-freedom viscous damping vibration system is shown in formula (20), where $A_{i}(t)=A_{0 i} e^{-\xi_{i} w_{n i} t} ; \mathrm{N}$ represents the modal order. $A_{0 i} 、 \mathcal{W}_{n i} 、 \mathcal{w}_{d i}$ and $\xi_{i}$ are respectively the $i$-th order amplitude, natural frequency of undamped vibration, natural frequency of damped vibration and damping ratio, and

$$
\begin{aligned}
& w_{d i}=\sqrt{1-\xi_{i}{ }^{2}} w_{n i} . \\
& x(t)=\sum_{i=1}^{N} A_{i}(t) \cos \left(w_{d} t+\varphi_{i}\right)
\end{aligned}
$$

The impulse response function of multi-degree-of-freedom viscous damping vibration system is subjected to wavelet transformation as formula (21).

$$
w_{x}(a, b)=\int_{-\infty}^{+\infty} \sum_{i=1}^{N} A_{\mathrm{i}}(t) \cos \left(w_{d} t+\varphi_{i}\right) \varphi_{a, b}^{*}(t) d t .
$$

Considering the linear nature of the wavelet transform, expanding the Taylor series $A(t)$ near $t=b$, and omitting the higher-order terms, it turns to formula (22). Decoupling the modes of each order, the wavelet transform expression of each independent mode after decoupling is shown in formula (23). 


$$
\begin{aligned}
& \mathcal{W}_{x}(a, b)=\sum_{i=1}^{i=N} \frac{\sqrt{a}}{2} A_{0 i} e^{j\left(w_{i d} b+\varphi_{i}\right)} \psi^{*}\left(a_{w_{i d}}\right) e^{-\xi w_{i n} b} . \\
& \mathcal{W}_{x}\left(a_{i}, b\right)=\frac{\sqrt{a_{i}}}{2} A_{0 i} e^{j\left(w_{i d} b+\varphi_{i}\right)} \psi^{*}\left(a_{\mathcal{W}_{i d}}\right) e^{-\xi_{i} w_{i n} b} \\
& \left\{\begin{array}{l}
\xi_{i} w_{i n}=-\frac{\mathrm{d}}{\mathrm{d} b}\left(\ln \left|w_{x}\left(a_{i}, b\right)\right|\right) \\
w_{i d}=\frac{\mathrm{d}}{\mathrm{d} b}\left(\arg \left(w_{x}\left(a_{i}, b\right)\right)\right)
\end{array}\right.
\end{aligned}
$$

To sum up, for a system with degrees of freedom, calculate the wavelet transform coefficient of the response function of each degree of freedom for the K-th degree of freedom at a certain time ( $b=b_{t}$ ) according to formula (23). Thus the $i$-th vibration mode of multi-degree-of-freedom system is obtained as formula (25). Different modes can be obtained by taking different values of $i$.

$$
\phi^{(i)}=\left[w_{h_{i}, 1}\left(a_{i}, b_{t}\right), \ldots \ldots, w_{h_{i}, k}\left(a_{i}, b_{t}\right), \ldots \ldots, w_{h_{i}, N}\left(a_{i}, b_{t}\right)\right]
$$

\section{High-speed Maglev Experiment Analysis}

The long-span beam is a typical representative of traditional structural system, and has many similarities with bridges. It is generally composed of beam body, bearing, buttress and foundation. The track beam used in high-speed maglev is called guideway. Due to the levitation characteristics of maglev transportation, such a guideway has high requirements for deformation and low tolerance for damage. Therefore, health monitoring and damage identification are of great significance. In this paper, an online real-vehicle vibration experiment study was conducted on the Shanghai high-speed maglev test line. In this study, 21 measuring points were arranged for a single-span track beam with a span of 12 meters for testing, including 14 vertical measuring points and 7 lateral measuring points. The acceleration time-domain curves of measuring points No.7(vertical), No.8(vertical), and No.18(lateral) are shown in Fig.1.

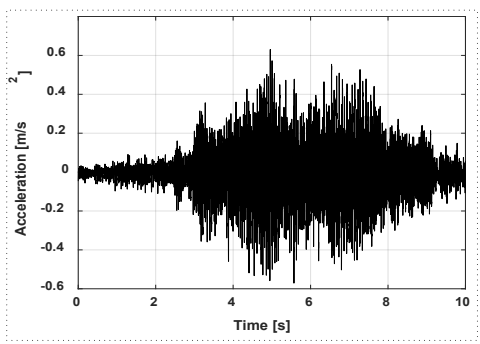

(a) Measuring point No.7(1024Hz)

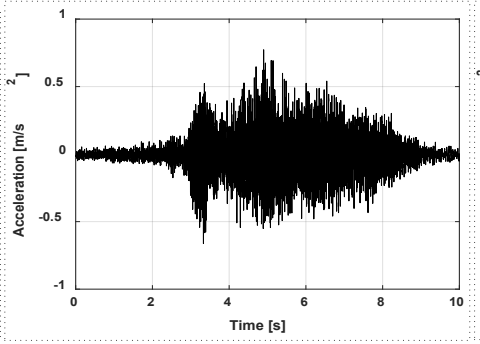

(b) Measuring point No.8(1024Hz)

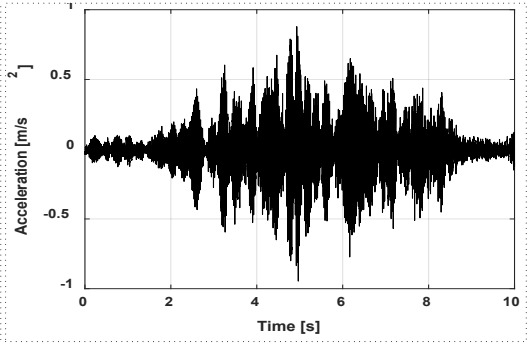

(c) Measuring point No.18(1024Hz)

Fig. 1 Time domain diagrams of vibration acceleration 
The three measuring points are all located near the middle span of the bottom of the guide rail. The response of the guideway excited by the maglev vehicle is obvious. The peak value of vertical acceleration is between $0.6-0.8 \mathrm{~m} / \mathrm{s}^{2}$, and the peak value of lateral acceleration is between $0.8-1.0 \mathrm{~m} / \mathrm{s}^{2}$. The mass of the vehicle is about $60 \mathrm{t}$, and the preliminary estimation of the acceleration time-domain curve is reasonable and effective.

In order to further verify the accuracy of the test data, this paper introduces the correlation function for comparative analysis to determine whether the measuring points or test equipment have the relevant coupling. The acceleration time-domain curves of measuring points No.7, No.8 and No.18 are real functions, and their correlation functions are shown in Fig.2.

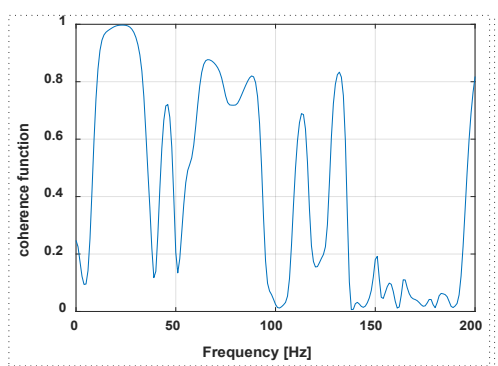

(a) Measuring point No.7 And No.8

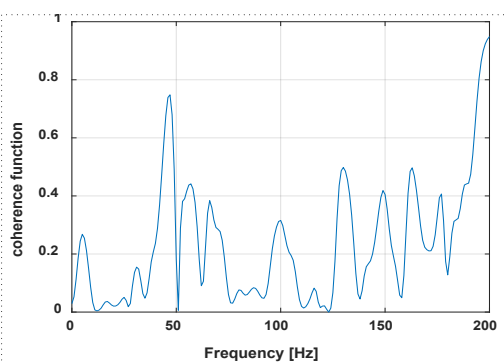

(b) Measuring point No.7 And No.18

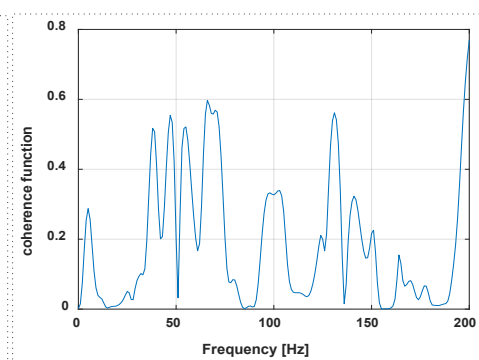

(c) Measuring point No.8 And No.18

Fig. 2 Correlation functions of measuring points

Comparing these functions, the correlation between measuring points No.7 and No.8 is relatively large. The two measuring points are located on both lateral sides of the same cross-section of the beam, and they are both vertical accelerations. Therefore, the correlation is quite high in the low frequency range. The guideway vibration is dominated by low-frequency vibration, which is concentrated below $100 \mathrm{~Hz}$. This is consistent with the measured data. The correlation between measuring points No.7 and No.8 clearly decreased, especially in the low frequency range. In the low frequency range, the correlation function is lower than 0.8 , which can be considered uncorrelated. It fully shows that the coupling degree of lateral vibration and vertical vibration of the guideway is relatively low, which is consistent with the theoretical analysis. Similarly, the correlation between measuring point No.8 and No.18 is also low.

The power spectrum represents the change of signal power with frequency in the unit frequency band, and reflects the distribution of signal power in the frequency domain. The shape of the power spectrum is without obvious burrs, and is relatively smooth, as shown in Fig. 3, which indirectly proves the accuracy of the experiment.

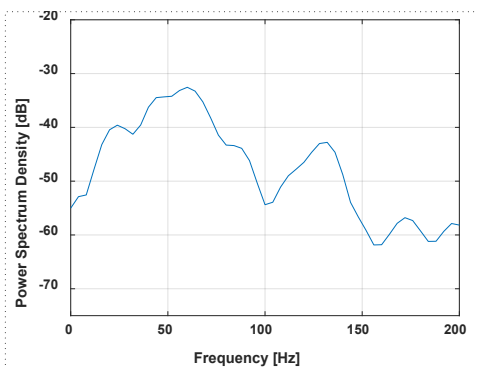

(a) Measuring point No.7

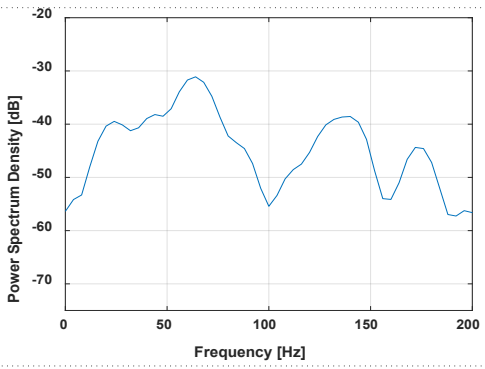

(b) Measuring point No.8

Fig. 3 Power spectrum density

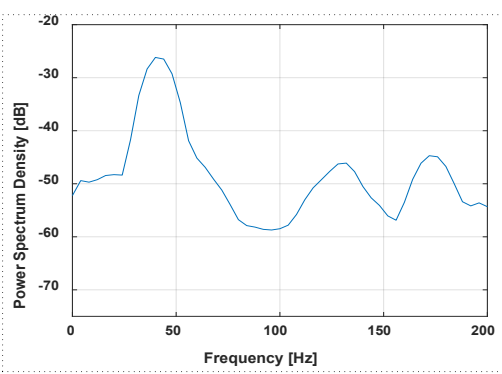

(c) Measuring point No.18 
The modal identification can be performed after Wavelet transform of the measuring point data. Take the measuring point No.7 and No.18 as an example, and the results of Wavelet transformation are shown in Fig.4.

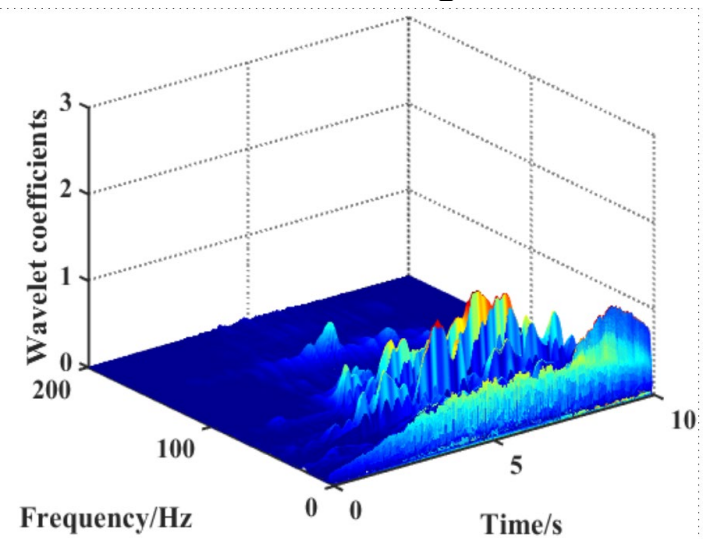

(a) Measuring point No.7

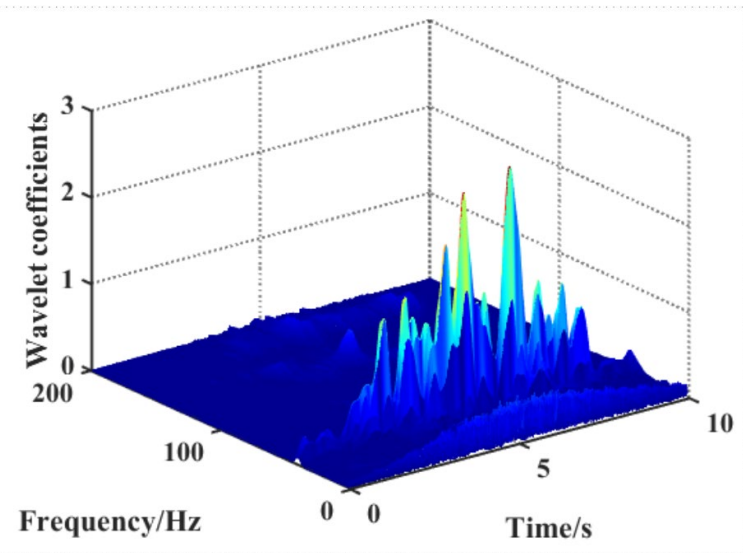

(b) Measuring point No.18

Fig. 4 Time domain and frequency domain comparison of Wavelet transform

According to the three-dimensional graph of the time, frequency, and Wavelet coefficients of the acceleration data of the measuring points, the vibration frequency of the measuring points is mainly concentrated in the low frequency below $100 \mathrm{~Hz}$. In other words, low frequency vibration is dominant.

\section{Conclusion}

The vibration test of Shanghai high-speed maglev test line fully reflects the vibration characteristics of the high-speed maglev guideway system after long-term use, for instance, the vibration frequency can be filtered by wavelet transform, and some vibration characteristics of guideways can be obtained. It is of particular significance for the following experiments and simulation model iterative updating, modal identification flaw detection and maglev guideways health monitoring experiment. According to the experimental analysis, it can be concluded that:

1) It is low that the coupling degree of vertical and lateral vibration of long-span high-speed maglev guideway. In the health monitoring experiment, it should be considered and compared respectively, and the specific quantitative correlation function is used to assist data processing and analysis;

2) By comparing the modal simulation and measured data of the high-speed maglev guideway, the vibration of the high-speed maglev guideway is mainly concentrated in the low-frequency band below $100 \mathrm{~Hz}$, and it is difficult for the operation of maglev vehicle to stimulate high-frequency vibration;

3) The lateral vibration of the high-speed maglev guideway is obvious, which will affect the lateral guide gap of high-speed maglev. Therefore, it can not be ignored in health monitoring, and vertical suspend gap and lateral guide gap should be considered comprehensively.

\section{References}

[1] Entezami A, Sarmadi H, Behkamal B, et al. Big Data Analytics and Structural Health Monitoring: A Statistical Pattern Recognition-Based Approach[J]. Sensors, 2020, 20(8):2328. https://doi.org/10.3390/s20082328 
[2] Yang J, Sha G, Zhou Y, et al. Statistical pattern recognition for structural health monitoring using ESN feature extraction method[J]. 2018. https://doi.org/10.2316/Journal.206.2018.6.206-4686

[3] Wang Q A, Ni Y Q. Measurement and Forecasting of High-Speed Rail Track Slab Deformation under Uncertain SHM Data Using Variational Heteroscedastic Gaussian Process[J]. Sensors, 2019, 19(15):3311. https://doi.org/10.3390/s19153311

[4] Liu X Z , Xu C, Ni Y Q. Wayside Detection of Wheel Minor Defects in High-Speed Trains by a Bayesian Blind Source Separation Method[J]. Sensors, 2019, 19(18):3981-. https://doi.org/10.3390/s19183981

[5] W.J. Staszewski, Identification of dampingin m.d.o.f systems usingtime-scale decomposition, Journal of Sound and Vibration. 1997(203). 283-305. https://doi.org/10.1006/jsvi.1996.0864

[6] M. Ruzzene, A. Fasana, L. Garibaldi, B. Piombo, Natural frequencies and dampings identification using wavelet transform: application to real data. Mechanical Systems and Signal Processing. 1997(2). 207-218. https://doi.org/10.1006/mssp.1996.0078

[7] P. Argoul, T. P. Le, Wavelet analysis of transient signals in civil engineering, Novel Approaches in Civil Engineering, Lecture Notes in Applied and Computational Mechanics, 2003(14), 200-213

[8] B. Boashash, P. O. Shea, Ploynormial Wigner-Ville distributions and their relationship to time-varying higher order spectra. IEEE trans. Signal Process. 1995(43): 216-220. https://doi.org/10.1109/78.258143

[9] B. Eschdi'e, B. Tor'esanni, Wavelet representation and time-scaled matched receiver for asymptotic signals. Proc. EUSIPCOV, Barcelona, 1990,305-308

[10] B.A.D. Piombo, A. Fasana, S. Marchesiello, et al. Modeling and identification of the dynamic response of a supported bridge. Mechanical Systems and Signal Processing, 2000,14(1): 75-89. https://doi.org/10.1006/mssp.1999.1266

[11] R.W.Baker, M.J.Hinich, Statistical monitoring of rotating machinery by cumulant spectral analysis. IEEE signal Processing workshop on Hinger-Order Statistic. 1993:187-191

[12] B.Boashash , Estimating the Instantaneous Frequency of a signal Part 1: Fundamentals. Proceedings of the IEEE, 1992,80(4), 520-538. https://doi.org/10.1109/5.135376

[13] W.J.Staszewski, Copper J.E. Flutter data analysis using the wavelet transform. Proceedings of the International Seminar on New Advances in Modal Synthesis of Large Structures: Nonlinear, Damped and Non-deterministic Cases, Lyon, France, 1995, 203-214

[14] Y.G.Wang,J.H.Zhang,The application of wavelet transform in dynamic system identification,Structure \& Environment Engineering1997(4) :22-29.

[15] I.Simonovski, M.Boltezar, The norms and variances of the Gabor, Morlet and General harmonic wavelet functions. Journal of Sound and Vibration. 2003(264): 545-557. https://doi.org/10.1016/S0022-460X(02)01206-3 